H. M. Colhoun - D. J. Betteridge · P. N. Durrington •

G. A. Hitman - H. A. W. Neil - S. J. Livingstone •

M. J. Thomason $\cdot$ J. H. Fuller $\cdot$ on behalf of the CARDS

Investigators

\title{
Rapid emergence of effect of atorvastatin on cardiovascular outcomes in the Collaborative Atorvastatin Diabetes Study (CARDS)
}

Received: 10 March 2005 / Accepted: 14 July 2005 / Published online: 12 November 2005

(C) Springer-Verlag 2005

\begin{abstract}
Aims/hypothesis: The aim of this study was to determine the pattern of the effect of the 3-hydroxy-3methylglutaryl coenzyme A reductase inhibitor atorvastatin on cardiovascular events in patients with type 2 diabetes and no prior history of cardiovascular disease (CVD). Materials and methods: A post hoc analysis of data from the Collaborative Atorvastatin Diabetes Study (CARDS), a randomised, placebo-controlled trial of 2,838 patients with type 2 diabetes, was performed. Patients received atorvastatin (10 mg daily) or placebo and were evaluated for car-
\end{abstract}

See http://www.cardstrial.org for a list of investigators

H. M. Colhoun $(\bowtie)$

The Conway Institute, University College Dublin,

Belfield,

Dublin 4, Ireland

e-mail: helen.colhoun@ucd.ie

Tel.: +353-1-7166951

Fax: $+353-1-7166950$

D. J. Betteridge

The Middlesex Hospital, University College London,

London, UK

P. N. Durrington

Division of Cardiovascular and Endocrine Science,

Department of Medicine, Manchester Royal Infirmary,

University of Manchester,

Manchester, UK

G. A. Hitman

Centre for Diabetes and Metabolic Medicine,

Barts and the London, Queen Mary's School

of Medicine and Dentistry,

London, UK

H. A. W. Neil

Oxford Centre for Diabetes, Endocrinology and Metabolism,

University of Oxford,

Oxford, UK

S. J. Livingstone $\cdot$ M. J. Thomason · J. H. Fuller

EURODIAB, Department of Epidemiology and Public Health,

Royal Free and University College Medical School,

London, UK diovascular and other outcomes over a median follow-up period of 3.9 years. Cox proportional hazards modelling was carried out, and the hazard ratios calculated for various times after randomisation to treatment were investigated. Results: A reduction in the primary endpoint of major CVD events was apparent and statistically significant as soon as 18 months after treatment initiation. The effect of atorvastatin on CHD events was apparent by 6 months, and at 1 year was similar to the $37 \%$ relative risk reduction observed at trial closure. Conclusions/interpretation: Atorvastatin alters the pathogenesis of CVD rapidly, such that the effect on cardiovascular events is apparent within months of initiation of therapy.

Keywords Cardiovascular - Diabetes $\cdot$ HMG-CoA reductase inhibitor · 3-Hydroxy-3-methylglutaryl coenzyme A reductase inhibitor

Abbreviations CARDS: Collaborative Atorvastatin Diabetes Study - CVD: Cardiovascular disease

\section{Introduction}

The Collaborative Atorvastatin Diabetes Study (CARDS) was a randomised, placebo-controlled trial comparing the effects of atorvastatin, $10 \mathrm{mg}$ daily, with those of placebo on major cardiovascular disease (CVD) in patients with type 2 diabetes and no prior CVD history. The main trial data have been published [1]; atorvastatin treatment was associated with a highly significant $37 \%$ reduction in the primary endpoint of major CVD events and with a $48 \%$ reduction in the incidence of stroke.

CARDS was terminated almost 2 years earlier than planned because at the second pre-scheduled interim analysis the data-safety-monitoring board found a highly significant benefit with treatment beyond that stipulated in the early stopping rule. Indeed, even at the first scheduled interim analysis the treatment effect was already significant at conventional levels $(p=0.03)$, although it was not suf- 
ficient to meet the stringent early stopping threshold $(p<0.001)$. This first interim analysis was triggered by the accrual of $25 \%$ of the total expected endpoints (76) in
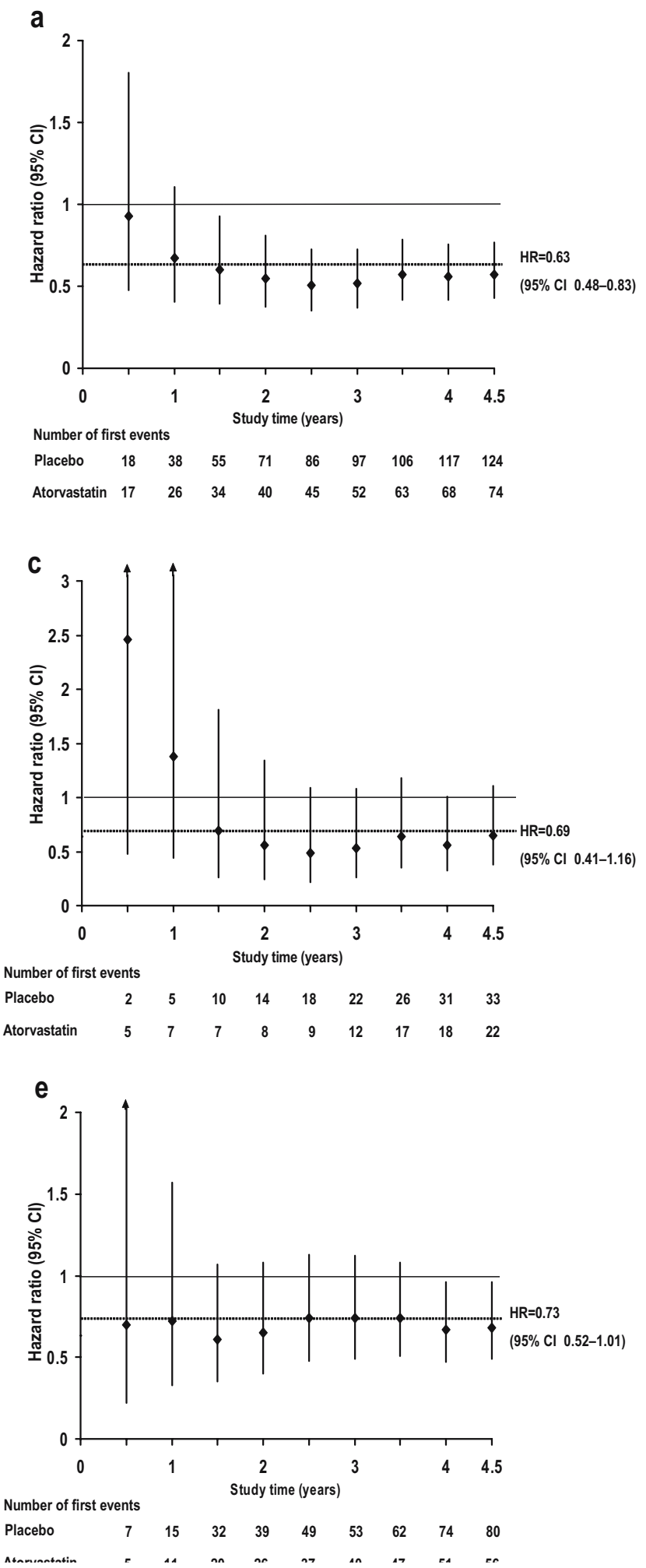

Fig. 1 Hazard ratios $(H R)$ for the effect of treatment with atorvastatin (10 $\mathrm{mg}$ daily) vs placebo on the following cardiovascular outcomes during follow-up: (a) major cardiovascular events, (b) CHD events, (c) revascularisation, (d) stroke and (e) all-cause
October 2000. The large treatment effect observed in CARDS was in part because the difference in LDL cholesterol between the active and placebo treatments was

b

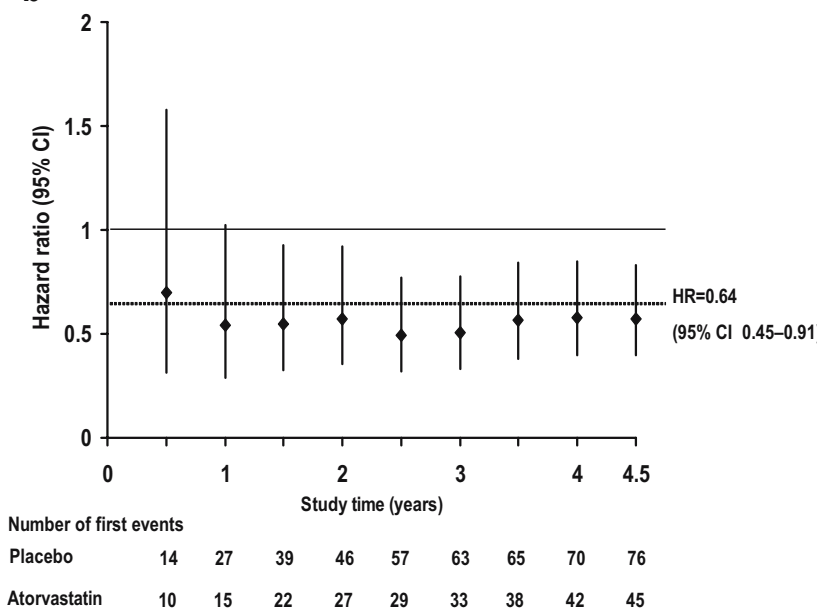

d

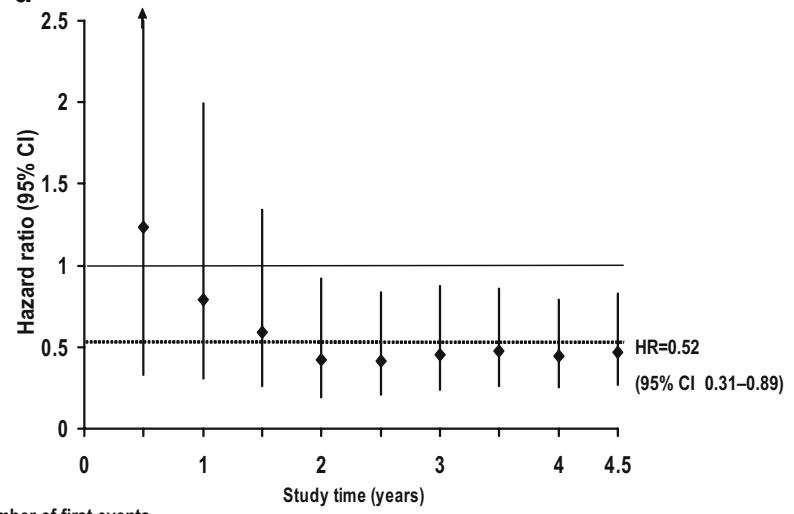

\begin{tabular}{rrrrrrrrrr} 
Number of first events & & & & & & & & \\
\hline & 4 & 10 & 15 & 21 & 26 & 28 & 33 & 37 & 37
\end{tabular}

$\begin{array}{llllllllll}\text { Atorvastatin } & 5 & 8 & 9 & 9 & 11 & 13 & 16 & 17 & 18\end{array}$ mortality. The solid lines show a hazard ratio of 1. Dashed lines show the actual final hazard ratio (i.e. treatment effect) across the study 
$-40 \%$ rather than the value of $-30 \%$ on which the power calculation had been based. Since the study was designed a meta-analysis has reported that a $37 \%$ reduction in LDL cholesterol is typically achieved with $10 \mathrm{mg}$ atorvastatin [2]. The median follow-up time in the trial at the time of termination was 3.9 years.

The purpose of this short report is to examine in more detail the treatment effect through time. We conducted a post hoc analysis of the hazard ratio associated with treatment for cardiovascular outcomes through time to assess whether there was a lag before atorvastatin treatment took effect, or whether the effect was present from early after treatment initiation.

\section{Materials and methods}

The detailed methods used in CARDS and the baseline characteristics of patients and the main outcomes have been published previously [1]. In brief, 2,838 patients with type 2 diabetes and no previous history of CVD were randomised to receive either placebo or $10 \mathrm{mg}$ atorvastatin daily. To be eligible for randomisation patients had to have, in addition to diabetes, at least one of the following risk factors: (1) a history of hypertension; (2) retinopathy (i.e. any retinopathy, maculopathy or prior photocoagulation); (3) micro- or macroalbuminuria; or (4) current smoker (all such patients were counselled to stop). Patients were ineligible if they had any past history of myocardial infarction, angina, coronary vascular surgery, cerebrovascular accident or severe peripheral vascular disease (defined as warranting surgery). The mean serum concentration of LDL cholesterol during baseline visits prior to randomisation had to be $\leq 4.14 \mathrm{mmol} /$ $1(160 \mathrm{mg} / \mathrm{dl})$ and that of serum triglycerides had to be $\leq 6.78 \mathrm{mmol} / 1(600 \mathrm{mg} / \mathrm{dl})$. Patients were excluded if during the placebo run-in phase they had less than $80 \%$ compliance with placebo. After randomisation patients were seen monthly for the first 3 months, at 6 months, and 6-monthly thereafter. Clinical cardiovascular events other than coronary revascularisations, silent myocardial infarctions and deaths were required to be acute and hospital-verified to be eligible as endpoints. An independent Endpoint Committee reviewed all reported cardiovascular events and all deaths and classified them according to criteria specified in the endpoint protocol. In addition to clinical events, annual ECGs were Minnesota-coded for the detection of any Qwave silent myocardial infarctions that had not presented clinically. Of the randomised subjects, $99.3 \%$ were fully evaluable for mortality and morbidity at study termination. The mean period of observation for the primary endpoint was 3.77 years (median 4.0) in the atorvastatin group and 3.66 years (median 3.9 ) in the placebo group.

CARDS was conducted in accordance with the Declaration of Helsinki and the Guidelines on Good Clinical Practice. Each centre involved in the study obtained Local Research Ethics Committee approval following approval from the Multicentre Research Ethics Committee. All patients gave fully informed, written consent.

\section{Statistical methods}

The primary endpoint comprised the first of the following; acute CHD event (i.e. myocardial infarction including silent infarction, unstable angina, acute CHD death, resuscitated cardiac arrest), coronary revascularisation procedures, or stroke. All analyses were by intention to treat. The main analysis in the present report is a Cox regression survival analysis comparing the hazard rates for the primary endpoint and its separate components in the two treatment groups and yielding hazard ratios as measures of effect size (treatment effect). The hazard ratios obtained by censoring the data at successively increasing time points are shown. The $p$ values are considered significant at conventional levels $(p<0.05)$ for effects other than those on the primary endpoint, for which $p<0.049$ was considered significant to allow for interim analysis effects on the type 1 error rate.

\section{Results}

As reported previously, at the completion of the trial there was a $37 \%$ reduction in the primary endpoint of major CVD events, a $36 \%$ reduction in acute coronary events, a $31 \%$ reduction in coronary revascularisation events and a $48 \%$ reduction in stroke. As shown in Fig. 1, by 1 year of follow-up the estimate of the treatment effect on the primary endpoint was already at its final value of a $37 \%$ reduction and by 18 months the CI did not include unity. For the acute CHD events considered, the point estimate of the treatment effect at 6 months of follow-up was similar to its final value and by 18 months the confidence limits did not include unity. For stroke, the treatment effect estimate was very unstable during the initial stages of the study, in keeping with the small number of events, but the treatment effect estimate at 18 months was at its final value and by 2 years the CI did not include unity. The total mortality rate was about $27 \%$ lower in the atorvastatin-treated group than in the placebo group for most of the follow-up period and was of borderline significance at termination.

\section{Discussion}

These data demonstrate that the beneficial effects of this low 10-mg dose of atorvastatin on CVD outcomes are already clear in this trial population by $12-18$ months. For CHD events it is already apparent at 6 months. These data are consistent with those from studies using other doses of atorvastatin in which the treatment benefits in patients with acute coronary syndromes were apparent within just weeks of initiation of atorvastatin treatment. In the PROVE IT study comparing the effect of atorvastatin, $80 \mathrm{mg}$ daily, with pravastatin, $40 \mathrm{mg}$ daily, in patients with a recent acute coronary event, by 30 days of follow-up the treatment effect estimate was similar to the overall trial estimate of $16 \%$ [3]. In the MIRACL study atorvastatin, $80 \mathrm{mg}$ daily, reduced the incidence of recurrent ischaemic events at 16 
weeks in patients with acute coronary syndromes [4]. In studies of other statins, such as simvastatin, treatment benefits have also emerged within the early years of the trial; in the Heart Protection Study for example among patients with diabetes, although not present at 1 year, a beneficial treatment effect was apparent at 2 years [5].

It is important to emphasise that this is a post hoc analysis and that its purpose is not to try to pinpoint a specific time at which the treatment suddenly starts to work. Indeed, it should be noted that as in many CVD endpoint trials, our pre-specified analysis plan was for a Cox proportional hazards analysis to estimate the treatment effect. Inherent in the choice of this model is the assumption, not often appreciated, that the hazards are proportional through time. In other words, this model assumes that the data are consistent with the same cumulative estimate of treatment effect being present throughout the trial, even in its very early stages, and therefore by implication that the treatment is effective immediately after its initiation. We confirmed that this proportionality assumption holds true in CARDS [1], but the plots presented here of the hazards ratio through time emphasise just how apparent that treatment effect is from an early stage. When designing trials of this nature and considering efficacy, lengthy follow-up could be reduced by increasing the sample size, because the treatment effect is not time-dependent. However, a reasonable time of followup is necessary to allow proper evaluation of safety. Where the treatment effect is neither time-dependent nor has a lag time, the point at which it becomes statistically significant is dependent only on the underlying placebo event rate and the magnitude of the treatment effect. Whilst, like most trials, CARDS was powered at $90 \%$, predicting a much later termination date, in reality there was substantial power to detect significant treatment effects earlier in the follow-up period, which was one of the purposes of specifying early stopping rules.

What is the mechanism underlying the early reduction in risk observed here with atorvastatin? An early treatment effect could be consistent with lipid-lowering alone leading to a reduction in plaque volume or to stabilisation of plaque by, for example, increasing the collagen to cholesterol ratio. In the REVERSAL trial Nissen et al. reported that a beneficial effect of atorvastatin ( $80 \mathrm{mg}$ daily) compared with pravastatin ( $40 \mathrm{mg}$ daily) on plaque volume in the coronary vessels was apparent using intravascular ultrasound by 18 months of follow-up [6]. Attention has been drawn recently to the potential importance of the lowering of C-reactive protein and possible inflammation reduction as a mechanism of atorvastatin action [7,8]. Whether such anti-inflammatory effects proposed for atorvastatin therapy are relevant to this early treatment effect has not yet been assessed in the CARDS data.
In conclusion, this post hoc analysis clearly demonstrates that atorvastatin therapy starts to effect a reduction in CVD from early after treatment initiation. This, and the high event rates for CVD in diabetes, emphasise that clinicians should not delay in assessing the needs of their patients with diabetes for such treatment.

Acknowledgements We are grateful to the patients who took part in this study, the study nurses and local investigators. This study was funded by the Department of Health UK, Diabetes UK and Pfizer.

Duality of interest The CARDS trial was partly funded by Pfizer Inc. H. Colhoun has received honoraria for acting as a consultant to Pfizer on their clinical trials advisory board. She has received speaker fees for giving lectures. J. H. Fuller is a member of a Pfizer clinical trials advisory board and has received remuneration for this activity and speaker fees for speaking at meetings. J. Betteridge is a member of a Pfizer clinical trials advisory board and has received remuneration for this activity. He has also received speaker fees for speaking at meetings. G. A. Hitman has received speaker fees from Pfizer for giving lectures. H. A. W. Neil is a member of a Pfizer diabetes advisory board and has received consultancy and speaker fees for giving lectures. P. N. Durrington is a member of a Pfizer clinical trials advisory board for which he has received remuneration. He has also spoken at meetings sponsored by Pfizer for which he has received speaker fees.

\section{References}

1. Colhoun HM, Betteridge DJ, Durrington PN et al (2004) Primary prevention of cardiovascular disease with atorvastatin in type 2 diabetes in the Collaborative Atorvastatin Diabetes Study (CARDS): multicentre randomised placebo-controlled trial. Lancet 364:685-696

2. Law MR, Wald NJ, Rudnicka AR (2003) Quantifying effect of statins on low density lipoprotein cholesterol, ischaemic heart disease, and stroke: systematic review and meta-analysis. BMJ 326:1423

3. Cannon CP, Braunwald E, McCabe CH et al (2004) Intensive versus moderate lipid lowering with statins after acute coronary syndromes. N Engl J Med 350:1495-1504

4. Schwartz GG, Olsson AG, Ezekowitz MD et al (2001) Effects of atorvastatin on early recurrent ischemic events in acute coronary syndromes: the MIRACL study: a randomized controlled trial. JAMA 285:1711-1718

5. Collins R, Armitage J, Parish S, Sleigh P, Peto R (2003) MRC/ BHF Heart Protection Study of cholesterol-lowering with simvastatin in 5963 people with diabetes: a randomised placebocontrolled trial. Lancet 361:2005-2016

6. Nissen SE, Tuzcu EM, Schoenhagen P et al (2004) Effect of intensive compared with moderate lipid-lowering therapy on progression of coronary atherosclerosis: a randomized controlled trial. JAMA 291:1071-1080

7. Nissen SE, Tuzcu EM, Schoenhagen P et al (2005) Statin therapy, LDL cholesterol, C-reactive protein, and coronary artery disease. N Engl J Med 352:29-38

8. Ridker PM, Cannon CP, Morrow D et al (2005) C-reactive protein levels and outcomes after statin therapy. $\mathrm{N}$ Engl J Med 352:20-28 